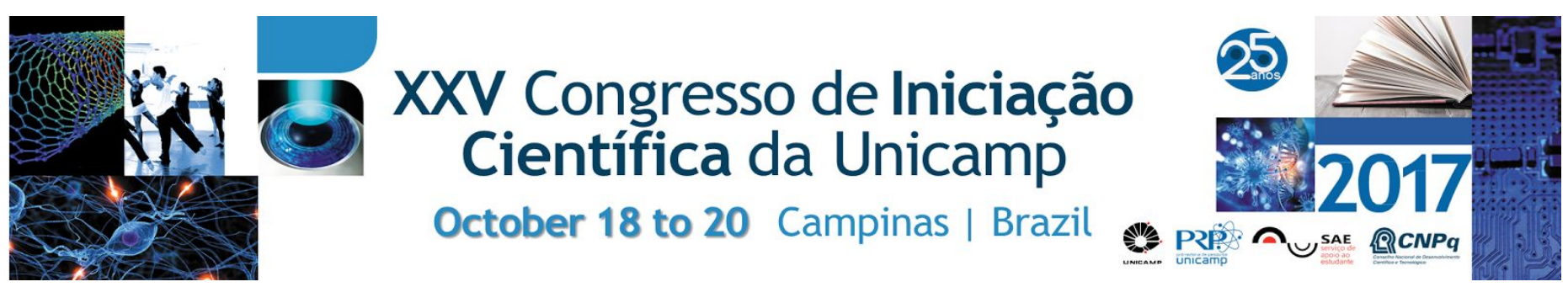

\title{
ASSESSMENT OF ONLINE TEACHING TOOLS FOR OCCASIONAL USE
}

\section{Levi Alves Gusmão de Faria*, Josué Labaki.}

\begin{abstract}
This work presents an assessment and selection criteria for online teaching platforms for short-term, non-regular lessons. Online platforms for occasional use differ from those meant for regular, long-term courses in that an occasional use might not justify their purchase or installation in the teacher's and the students' devices, for example. The aim of this project was to find as many available platforms as possible, and to rank them in terms of criteria established by the University of Campinas' academic community, which are their end users, and target audience of this project. A list of the ranked platforms, together with a flow chart to help teachers select the best available platform for their specific practice, as well as a description and tutorial of the top-ranked platform, was published at Unicamp's website.
\end{abstract}

\section{Key words:}

Online teaching, platform review, remote.

\section{Introduction}

Online platforms for occasional use differ from those meant for regular, long-term courses in that an occasional use might not justify their purchase or installation in the teacher's and the students' devices, for example. There are many available tools, which can be free or paid, require or not its installation, be suitable or not for mobile devices, enable or not live video and real-time feedback, and so on. The aim of this project was to find as many available platforms as possible, and to rank them in terms of criteria established by the University of Campinas' academic community, which are their end users, and target audience of this project.

\section{Results and Discussion}

In the initial assessment part of the project, a list of 40 platforms was found. Each platform was characterized by 10 features such as being free or paid, enabling or not live video, etc. An online survey was created and distributed for a statistically significant sample of the academic community of Unicamp to vote, so each feature could be ranked according to its importance in their teaching practice. At the end, all 40 platforms were ranked according to these criteria. A list of the ranked platforms, together with a flow chart to help teachers select the best available platform for their specific practice, as well as a description and tutorial of the top-ranked platform, was published at the official site of the University of Campinas.

\section{Conclusions}

At the end of the project, we could rank all the platforms analysed, and the top 10 positions were formed by commercial and open code softwares. The first position of our list consist of an open source software called Apache OpenMeetings $₫$. A tutorial to help the community to choose the best alternative of platform was created and made available in the project's site.

\section{Acknowledgement}

This project was supported by PAPI-SAE Scholarship, with resources provided by the University of Campinas. 